This item was submitted to Loughborough's Research Repository by the author.

Items in Figshare are protected by copyright, with all rights reserved, unless otherwise indicated.

\title{
Bridging the terahertz gap for chaotic sources with superconducting junctions
}

PLEASE CITE THE PUBLISHED VERSION

https://doi.org/10.1103/physrevb.99.060501

\section{PUBLISHER}

(C) American Physical Society (APS)

VERSION

VoR (Version of Record)

\section{PUBLISHER STATEMENT}

This work is made available according to the conditions of the Creative Commons Attribution-NonCommercialNoDerivatives 4.0 International (CC BY-NC-ND 4.0) licence. Full details of this licence are available at: https://creativecommons.org/licenses/by-nc-nd/4.0/

\section{LICENCE}

CC BY-NC-ND 4.0

\section{REPOSITORY RECORD}

Gulevich, D, V.P. Koshelets, and Feodor Kusmartsev. 2019. "Bridging the Terahertz Gap for Chaotic Sources with Superconducting Junctions". figshare. https://hdl.handle.net/2134/36901. 


\title{
Bridging the terahertz gap for chaotic sources with superconducting junctions
}

\author{
D. R. Gulevich, ${ }^{1}$ V. P. Koshelets, ${ }^{2}$ and F. V. Kusmartsev ${ }^{3,4,1, *}$ \\ ${ }^{1}$ ITMO University, St. Petersburg 197101, Russia \\ ${ }^{2}$ Kotelnikov Institute of Radio Engineering and Electronics, Russian Academy of Science, Moscow 125009, Russia \\ ${ }^{3}$ Micro/Nano Fabrication Laboratory Microsystem and THz Research Center, Chengdu, Sichuan 610200, China \\ ${ }^{4}$ Department of Physics, Loughborough University, Loughborough LE11 3TU, United Kingdom
}

(Received 24 January 2018; revised manuscript received 23 August 2018; published 5 February 2019)

\begin{abstract}
We observe a broadband chaotic signal of terahertz frequency emitted from a superconducting junction. The generated radiation has a wide spectrum reaching $0.7 \mathrm{THz}$ and power sufficient to drive on-chip circuit elements. Our experimental finding is fully confirmed by numerical modeling based on microscopic theory and reveals the unrealized potential of superconducting systems in chaos-based terahertz communication, fast generation of true random numbers, and noninvasive terahertz spectroscopy.
\end{abstract}

DOI: 10.1103/PhysRevB.99.060501

Since the call for bridging the terahertz gap has grown $[1,2]$, many physical systems have been proposed as sources of terahertz radiation (from 0.3 to $3 \mathrm{THz}$ ). The years of intense research have yielded quantum cascade lasers [3,4], semiconductor lasers and transistors [5-8], Gunn diodes [9], frequency multipliers [10], and generators based on low and high temperature superconductors [11-24]. With the terahertz gap for radiation sources gradually filling up with new practical and compact devices, there is yet one more terahertz gap to fill: the one for chaotic radiation sources. The need for them arises in cryptography which uses chaos to encrypt information [25-27], high-resolution $\mathrm{THz}$ spectroscopy [28$31]$, and computer engineering which demands high-rate true random number generators [32]. Although alternative technologies for generation of true random numbers exist, which rely on quantum effects [33-35] and various entropy sources [36-38], the use of chaos-based systems allows to achieve higher generation rates [39-42].

Chaos in superconducting systems first attracted attention of the scientific community when chaotic solutions of the driven sine-Gordon model describing the small Josephson junction (SJJ) under rf radiation were found [43]. While the early studies of chaos in Josephson junctions [44-46] were primarily centered around the rf-driven single SJJ, the more recent ones focused on the origins of the structured chaotic behavior of the SJJ [47], as well as several coupled SJJ oscillators [48-51]. Despite progress in achieving a chaotic state of extended Josephson systems [52], all existing experimental studies of chaos in superconducting systems so far dealt with microwave frequencies $(<100 \mathrm{GHz})$, which are significantly below the interest of modern terahertz science.

In this Rapid Communication, we present our results for generation of high-frequency broadband chaos reaching $0.7 \mathrm{THz}$ in frequency. A highly chaotic regime is achieved in a T-shaped Josephson junction which we here refer to as the T-junction flux-flow oscillator (TFFO); see Fig. 1. In contrast

*Corresponding author: F.Kusmartsev@lboro.ac.uk to the conventional flux-flow oscillator (FFO) [53] consisting of a tapered Josephson transmission line [54], the TFFO is made of two perpendicular lines coupled via a $\mathrm{T}$ junction: the main (MJTL) and additional (AJTL) Josephson transmission lines. While it has been understood in the past that the $T$ junction brings new effects to the dynamics of a single fluxon by slicing it into pieces [55-58], introducing the T junction in a standard FFO yields a completely unexpected result: it transforms one of the narrowest linewidth source ever created [17] to the opposite extreme: a highly chaotic generator with a very broad radiation spectrum. Indeed, results that we present in this Rapid Communication demonstrate the onset of chaos in almost all of the current-voltage characteristics (IVCs) of the device which includes the region of the terahertz gap $0.3-0.9 \mathrm{THz}$ where compact and practical devices are highly wanted.

High-frequency chaos generators promise to impact several independent areas of research and technologies. The high data rates of THz-frequency chaotic signals will make viable chaos-based communications. Development of $\mathrm{THz}$ chaotic sources will open opportunities for the chaos-based molecular and biological spectroscopy where sources of high-frequency broadband noise are needed [29]. The resulting chaos-based $\mathrm{THz}$ spectroscopy can be used to probe physical and chemical processes in biological and living systems which otherwise would be impossible or extremely difficult to observe using the tools of the conventional spectroscopy: transient biological structures, unstable molecules, and chemical reactions.

The TFFO is very different from all existing superconducting systems. Most notably, the electric current oscillations in the TFFO do not occur at the Josephson frequency $\left(f_{V}=\right.$ $2 e V / h$, which varies with the applied voltage $V$ ). Moreover, in some regimes, the TFFO frequency spectrum of Josephson current oscillations does not even peak at the Josephson frequency. Because the coupling between the tunnel currents and electromagnetic waves is not at a single frequency, but involves all possible modes of the whole spectrum, this creates tremendous theoretical difficulties which cannot be resolved with the standard approaches $[59,60]$. 


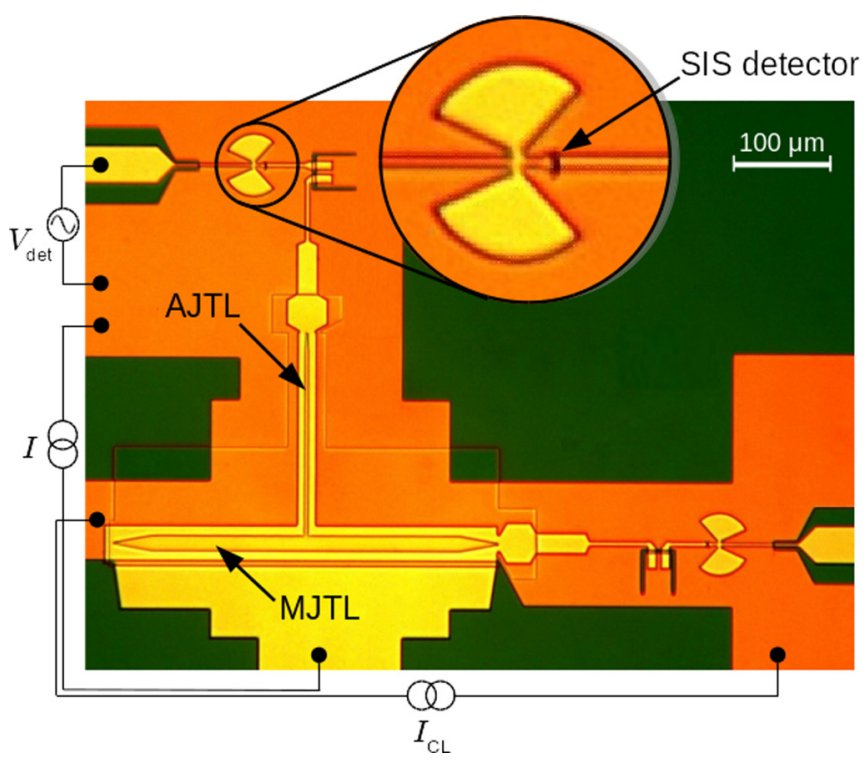

FIG. 1. Optical microphotograph of the experimental sample of the T-junction flux-flow oscillator (TFFO) fabricated using the Nb-AlN-NbN technology $[61,62]$. The TFFO consists of the additional Josephson transmission line (AJTL) coupled to the main Josephson transmission line (MJTL) in the form of a T junction. The TFFO is coupled to two SIS detectors, one at the AJTL and the other at the MJTL ends. Zoom of the SIS detector coupled to the AJTL is shown in the inset.

Several samples of the TFFO (one of them presented in Fig. 1) were fabricated using the Nb-AlN-NbN technology $[61,62]$ which allows us to achieve high radiation frequencies [63]. A control sample of the standard FFO with length equal to the length of the MJTL in the TFFO $(400 \mu \mathrm{m})$ has been fabricated on the same chip for testing the setup.

The IVC of a standard FFO consists of a set of curves which correspond to a fixed value of the electric current in a control line $I_{\mathrm{CL}}$ used to induce the external magnetic field. In the region of moderate voltages [up to $1.2 \mathrm{mV}$ in Fig. 2(a)] FFO exhibits a series of Fiske steps (FSs) arising when the standing electromagnetic waves in the junction resonate with the rate at which fluxons enter and leave the junction. Typically, for junctions of moderate lengths in the range 200$600 \mu \mathrm{m}, \mathrm{FSs}$ are pronounced up to the voltage $V_{g} / 3[64,65]$ ( $V_{g}$ is the gap voltage) where an abrupt increase of the damping suppresses propagation of electromagnetic modes. It occurs due to the phenomenon of self-coupling when the tunneling of quasiparticles through the barrier is enhanced by absorption of photons generated by the junction [66]. The radiation emitted from the FFO is detected by a superconductorinsulator-superconductor junction (SIS). The IVC of the SIS irradiated by the FFO exhibits a series of sharp and prominent Shapiro and quasiparticle steps which correspond exactly to the Josephson frequency at which the FFO is driven. The SIS can also be operated as a mixer by mixing the signals from the FFO and from a local oscillator.

In contrast, the IVC of the TFFO is drastically different; see Fig. 2(b). A striking feature is a nonzero return current $I_{\min }$ which can be interpreted as the presence of a finite barrier for fluxons passing through the $\mathrm{T}$ junction. Moreover, all curves exhibit a remarkable suppression of FSs and appear smooth even below the voltage $V_{g} / 3$ where the standard FFO would exhibit pronounced steps [cf. Fig. 2(a)]. The observed suppression of FSs is understood as follows. In general, the presence of a step on the IVC depends on whether a standing electromagnetic wave may form, i.e., whether the traveling electromagnetic wave can propagate from one end to the other without significantly losing its energy, and whether there is a regularity in fluxon dynamics, i.e., fluxons enter and leave the junction at regular time intervals. In our system, while the first condition is satisfied as suggested by the study of the control FFO sample of the same length, it is the second one being violated and signifies a very irregular fluxon dynamics. The noted irregularity of fluxon dynamics is further confirmed by the measurements of TFFO radiation using the two SIS detectors (see Fig. 2).

IVCs of SIS detectors operated in the power detection mode reveal very smooth quasiparticle steps and suggest broad radiation spectrum. The shapes of the quasiparticle steps of the AJTL SIS detector [the upper inset to Fig. 2(b)] suggest that the spectrum radiated from the AJTL is at least $0.1 \mathrm{THz}$ as broad. Even more surprisingly, quasiparticle steps on the IVC of the AJTL SIS are independent of the Josephson frequency $f_{V}$ (see also our results for other TFFO samples in Ref. [67]).

The spectrum radiated from the MJTL and AJTL ends (see Fig. 1) was analyzed by both SISs operated in the mixer mode. Although, the maximal bandwidth of the down-converted spectrum was limited from above by $1 \mathrm{GHz}$, we managed to scan this frequency window over the whole frequency range. A broad line has been identified in the spectrum of MJTL radiation in the high-voltage and high-magnetic-field region of the IVC [see the inset to Fig. 2(b)]: this was the narrowest line we could detect from the TFFO as its linewidth increases rapidly with decreasing applied voltage. No detectable peak at all was observed in the spectrum of AJTL radiation-rather, a featureless flat noise level within the $1 \mathrm{GHz}$ bandwidth. This agrees with our measurement of SIS detectors operated in the power detection regime presented above.

Our measurements of the TFFO radiation by both SIS detectors operated in the power detection and mixing modes, supported by the absence of FSs, indicate a very chaotic nature of the emitted radiation. We have found that the chaotic spectrum from the AJTL persists on all the IVCs, all the way up to the high-frequency region near the $\mathrm{Nb}$ superconducting gap frequency (about $0.7 \mathrm{THz}$ ) where the onset of damping hinders operation of the device.

Standard theoretical models of long Josephson junctions based on the the perturbative sine-Gordon equation take into account the self-coupling as a phenomenological modification of the damping parameter assuming the spectrum is dominated by a single harmonics at the Josephson frequency $[59,60]$. It is evident that while such approach is justified in the case of a standard FFO exhibiting a narrow radiation linewidth, it would a priori fail in the description of the TFFO because of a large number of competing electromagnetic modes as suggested by the broad spectrum observed in our experiment. In this case, coupling between the generated radiation and tunnel currents occurs for many excited modes of the chaotic spectrum simultaneously. We 

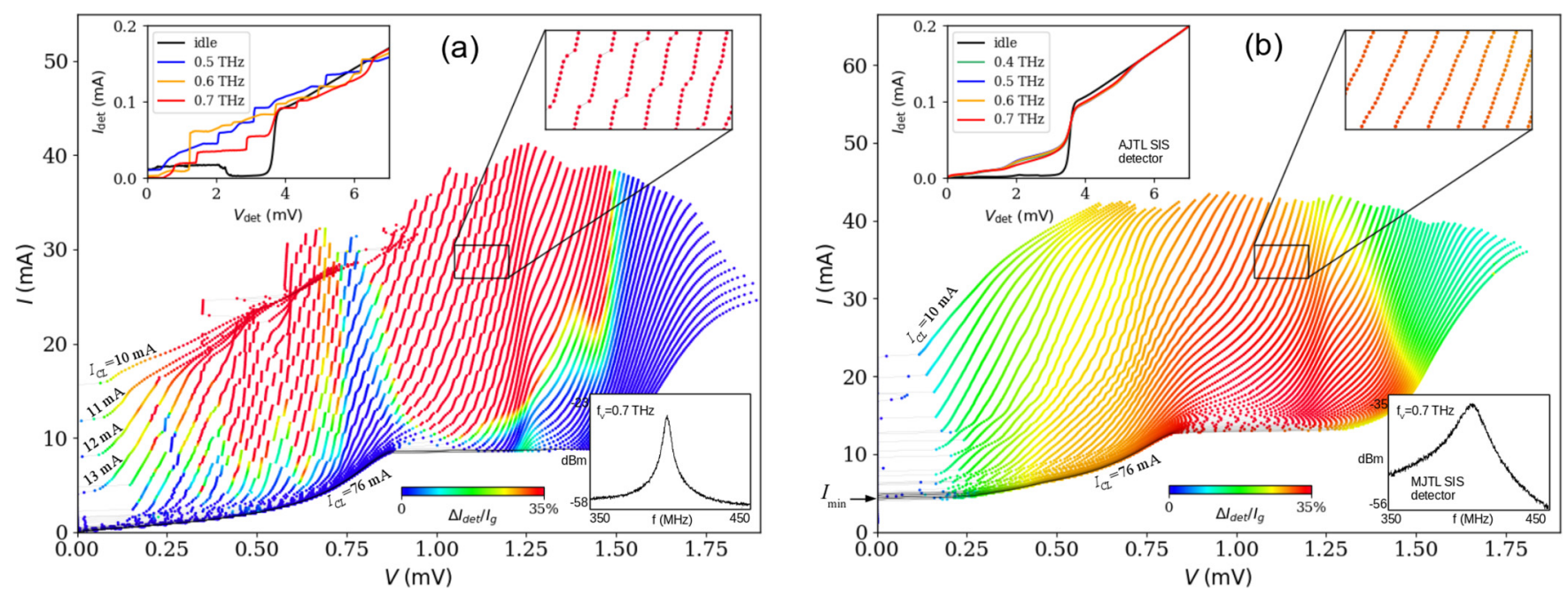

FIG. 2. (a) Current-voltage characteristics (IVCs) of the control sample: FFO of a standard layout. A set of IVC curves is measured at different values of the control line current $I_{\mathrm{CL}}$ ranging from $10 \mathrm{~mA}$ (the leftmost curve) to $76 \mathrm{~mA}$ (the rightmost curve) with an increment of $1 \mathrm{~mA}$. The color scale corresponds to the rise $\Delta I_{\text {det }}$ in the dc current through the SIS detector from 0 to $35 \%$ of the full step $I_{g}$ at the gap voltage (the precise definition of $I_{g}$ is given in Ref. [68]). The data where dc current rises above the $35 \%$ threshold are painted by the same (red) color as the $35 \%$ rise. Note that Fiske steps are pronounced up to the boundary voltage $V_{g} / 3$ (about $1.2 \mathrm{mV}$ ). Response of the SIS detector to the radiation emitted by the FFO is shown on the inset. The black line corresponds to an autonomous operation of the SIS detector; the blue, orange, and red lines represent pumping by the FFO at voltage $V$ specified in the legend by the Josephson frequency $f_{V}=2 e V / h$. Note the presence of sharp Shapiro steps in the IVCs of the detector and quasiparticle steps whose positions correspond exactly to the Josephson frequency of the FFO. (b) IVC of one of the fabricated experimental samples of the TFFO. As compared to the standard FFO, the Fiske steps are practically absent while the IVC curves are very smooth even below $V_{g} / 3$. The inset shows IVCs of the SIS detector coupled to the AJTL end taken at different values of TFFO voltage. Note that the SIS detector is driven not on the Josephson frequency but instead exhibits very broad steps centered at approximately the same voltage. The inset on the bottom right of the panel (b) shows the line profile measured from the MJTL at the Josephson frequency $f_{V}=0.7 \mathrm{THz}$. The red and blue lines correspond to the free-running and phase-locked regimes, respectively. This is about the narrowest radiation line we could measure from the TFFO. The linewidth increases rapidly with decreasing frequency below $0.7 \mathrm{THz}$. No detectable line was measured for radiation from the AJTL at all, but a featureless flat noise level. For comparison, the line profile for the standard FFO is shown in the inset at the right of panel (a).

term such dynamical regime chaotic self-coupling in contrast to conventional self-coupling when only coupling to the single electromagnetic mode at $f_{V}$ is relevant.

To obtain the theoretical treatment of the TFFO in the regime of the chaotic self-coupling it is, therefore, essential to account for coupling between the quasiparticle tunnel currents and electromagnetic field oscillations of all the involved frequencies. Such description is natural within the microscopic tunneling theory (MTT) of Josephson junctions [66,69-71]. Application of MTT to large Josephson junctions has been recently pushed forward by the authors in Refs. [72,73] and motivated the development of MiTMoJCo code [74,75]. We used the deal.II finite element library [76-78] in conjunction with MiTMoJCo to solve the integro-differential equation describing the two-dimensional model of the TFFO. The two-dimensional mesh was generated using the Gmsh mesh generator [79]. For evaluation of the tunnel currents we used smoothed tunnel current amplitudes calculated from the Bardeen-Cooper-Schrieffer theory for the $\mathrm{Nb}-\mathrm{AlN}-\mathrm{NbN}$ junction made of superconductors with different energy gaps: $\mathrm{Nb}(1.4 \mathrm{meV})$ and $\mathrm{NbN}(2.3 \mathrm{meV})$ at $T=4.2 \mathrm{~K}$ (see Ref. [67] for further details).

Results of our numerical calculations are presented in Fig. 3. The IVC curves were obtained at different values of the parameter $h_{\text {ext }}$ which describes the external magnetic field induced by the control line current $I_{\mathrm{CL}}$ in our experimental setup. The numerical IVC exhibits the same features as the experimental IVC of the TFFO: a nonzero return current $I_{\text {min }}$ and the absence of FSs. We have checked that the suppression of the FSs is not caused by damping by running a simulation for the standard FFO of the same length and observing wellpronounced FSs below $V_{g} / 3$. To reliably conclude about the chaotic regime we calculated the maximal Lyapunov exponent $\lambda_{\max }$ at every point of the numerical IVC of the TFFO using the procedure of Benettin et al. [80] generalized to the presence of memory [81]. The results of our calculation are presented Fig. 3(a). As seen from the figure, the maximal Lyapunov exponent $\lambda_{\max }$ takes positive values in the whole IVC apart from the zero-voltage state. The numerically calculated power spectra of the TFFO radiation are shown in Figs. 3(b) and 3(c). In full agreement with our experiments, the spectrum of radiation from the MJTL, while being very broad, shows some dependence on the Josephson frequency $f_{V}$, whereas the spectrum emitted from the AJTL does not even peak at the Josephson frequency.

To analyze the transition to chaos in our numerical simulations we introduced a control parameter $h_{X}$ which can be interpreted as a magnetic field component applied along the horizontal axis in Fig. 1 . When $h_{X}=0$, the TFFO exhibits a chaotic regime as has been demonstrated above. The gradual increase of $h_{X}$ forces the system to enter a regime of periodic oscillations at $h_{X} \sim h_{\mathrm{ext}}$. This signifies that it 

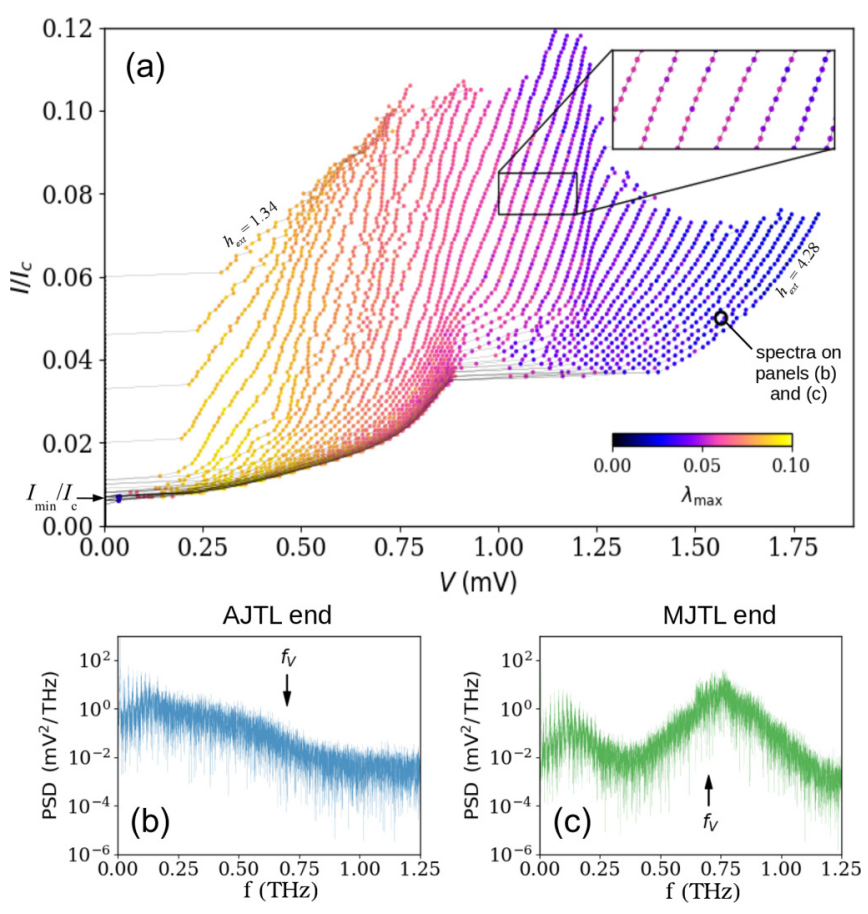

FIG. 3. (a) Theoretical current-voltage characteristic (IVC) of the T-junction flux-flow oscillator (TFFO) calculated using the finite element library deal.II [76] in conjunction with the microscopic tunneling library MiTMoJCo $[74,75]$. The curves are obtained at different values of the external magnetic field $h_{\text {ext }}$ changing from 1.34 to 4.28 with an increment 0.07 in normalized units (see Ref. [67] for details). The color scale represents the numerically calculated maximal Lyapunov exponent $\lambda_{\max }$ which indicates the presence of chaotic dynamics at every point of the flux-flow IVC. Panels (b) and (c) display the chaotic spectra at the AJTL and MJTL ends at the Josephson frequency $f_{V}=0.7 \mathrm{THz}$. The corresponding point on the IVC is marked by a black circle in (a).

is the dilute mixture of weakly bound fluxons in the AJTL which is responsible for the observed chaos. Indeed, as long as fluxons in the AJTL are arranged into a dense chain at high $h_{X}$, regular oscillations are observed, whereas when fluxons become diluted at smaller $h_{X}$ the chaotic dynamics gradually builds up. Our analysis shows that with decreasing $h_{X}$ the system undergoes the Ruelle-Takens-Newhouse route to chaos [82] by sequential torus splitting from a limit circle in the phase space to a $2 \mathrm{D}$ and then $3 \mathrm{D}$ torus which precede the transition to chaos.

To conclude, we detected a terahertz-range chaotic signal from a superconducting Josephson junction. As this is the first such experimental observation of a chaotic signal emitted from a superconducting system in this frequency range, this finding presents a candidate for filling the terahertz gap for chaotic oscillators. The practical potential of the TFFO chaotic generators is favored by the well-established $\mathrm{Nb}$ technology. It is routinely used for fabrication of standard superconducting junctions which have established their reputation as reliable $\mathrm{THz}$ sources for spectral measurements both in the laboratory $[23,83]$ and in the field $[84,85]$.

Our results suggest that the range of possible applications of superconducting junctions is far beyond their present use: it should include devices for chaos-based communications and generators of random numbers actively searched for in various alternative technologies. The proposed mechanism for generation of chaos opens horizons and opportunities for developing noise-based molecular and biological spectroscopy [29]. The novel chaos-based $\mathrm{THz}$ spectroscopy can be used to probe physical and chemical processes in biological and living systems which otherwise would be impossible or extremely difficult to observe. Broadband chaotic THz irradiation will enable us to obtain a noninvasive spectral snapshot of an undisturbed system, which otherwise would be impossible to obtain with the tools of conventional spectroscopy. In contrast to the tools of conventional spectroscopy, the chaos-based spectroscopy may display a whole range of absorption lines simultaneously providing instantaneous radiation spectra in fast biological processes, transient biological structures, unstable molecules, radicals, and chemical reactions.

The theoretical part of the work and numerical modeling are supported by the Russian Science Foundation under Grant No. 18-12-00429. The experimental study is supported from Grant No. 17-52-12051 of the Russian Foundation for Basic Research.
[1] B. Ferguson and X.-C. Zhang, Nat. Mater. 1, 26 (2002).

[2] P. H. Siegel, IEEE Trans. Microw. Theory Tech. 50, 910 (2002).

[3] R. Köhler, A. Tredicucci, F. Beltram, H. E. Beere, E. H. Linfield, A. G. Davies, D. A. Ritchie, R. C. Iotti, and F. Rossi, Nature (London) 417, 156 (2002).

[4] B. S. Williams, Nat. Photonics 1, 517 (2007).

[5] H. W. Hübers, S. G. Pavlov, and V. N. Shastin, Semicond. Sci. Technol. 20, S211 (2005).

[6] Y. Chassagneux, R. Colombelli, W. Maineult, S. Barbieri, H. E. Beere, D. A. Ritchie, S. P. Khanna, E. H. Linfield, and A. G. Davies, Nature (London) 457, 174 (2009).

[7] J. Lusakowski, W. Knap, N. Dyakonova, L. Varani, J. Mateos, T. Gonzalez, Y. Roelens, S. Bollaert,
A. Cappy, and K. Karpierz, J. Appl. Phys. 97, 064307 (2005).

[8] P. Li, Y. C. Wang, and J. Z. Zhang, Opt Exp. 18, 20360 (2010).

[9] S. Pérez, T. González, D. Pardo, and J. Mateos, J. Appl. Phys. 103, 094516 (2008).

[10] A. Maestrini, J. S. Ward, J. J. Gill, C. Lee, B. Thomas, R. H. Lin, G. Chattopadhyay, and I. Mehdi, IEEE Trans. Microwave Theory Technol. 58, 1925 (2010).

[11] A. K. Jain, K. K. Likharev, J. E. Lukens, and J. E. Sauvageau, Phys. Rep. 109, 309 (1984).

[12] S. Han, B. Bi, W. Zhang, and J. E. Lukens, Appl. Phys. Lett. 64, 1424 (1994).

[13] P. A. A. Booi and S. P. Benz, Appl. Phys. Lett. 68, 3799 (1996). 
[14] R. P. Robertazzi and R. A. Buhrman, Appl. Phys. Lett. 53, 2441 (1988).

[15] M. Darula, T. Doderer, and S. Beuven, Supercond. Sci. Technol. 12, R1 (1999).

[16] P. Barbara, A. B. Cawthorne, S. V. Shitov, and C. J. Lobb, Phys. Rev. Lett. 82, 1963 (1999).

[17] V. P. Koshelets and S. V. Shitov, Supercond. Sci. Technol. 13, R53 (2000).

[18] M. Tarasov, L. Kuz'min, E. Stepantsov, I. Agulo, A. Kalabukhov, M. Fominskii, Z. Ivanov, and T. Claeson, JETP Lett. 79, 298 (2004).

[19] L. Ozyuzer, A. E. Koshelev, C. Kurter, N. Gopalsami, Q. Li, M. Tachiki, K. Kadowaki, T. Yamamoto, H. Minami, H. Yamaguchi, T. Tachiki, K. E. Gray, W.-K. Kwok, and U. Welp, Science 318, 1291 (2007).

[20] H. B. Wang, S. Guénon, B. Gross, J. Yuan, Z. G. Jiang, Y. Y. Zhong, M. Grünzweig, A. Iishi, P. H. Wu, T. Hatano, D. Koelle, and R. Kleiner, Phys. Rev. Lett. 105, 057002 (2010).

[21] T. Kashiwagi, M. Tsujimoto, T. Yamamoto, H. Minami, K. Yamaki, K. Delfanazari, K. Deguchi, N. Orita, T. Koike, R. Nakayama, T. Kitamura, M. Sawamura, S. Hagino, K. Ishida, K. Ivanovic, H. Asai, M. Tachiki, R. A. Klemm, and K. Kadowaki, Jpn. J. Appl. Phys. 51, 010113 (2012).

[22] U. Welp, K. Kadowaki, and R. Kleiner, Nat. Photonics 7, 702 (2013).

[23] V. P. Koshelets, P. N. Dmitriev, M. I. Faley, L. V. Filippenko, K. V. Kalashnikov, N. V. Kinev, O. S. Kiselev, A. A. Artanov, K. I. Rudakov, A. de Lange, G. de Lange, V. L. Vaks, M. Y. Li, and H. B. Wang, IEEE Trans. Terahertz Sci. Technol. 5, 687 (2015).

[24] F. Boussaha, C. Chaumont, A. Féret, J-G. Caputo, and T. Vacelet, IEEE Trans. Appl. Supercond. 26, 1501405 (2016).

[25] S. Hayes, C. Grebogi, and E. Ott, Phys. Rev. Lett. 70, 3031 (1993).

[26] A. Argyris, D. Syvridis, L. Larger, V. Annovazzi-Lodi, P. Colet, I. Fischer, J. García-Ojalvo, C. R. Mirasso, L. Pesquera, and K. Alan Shore, Nature (London) 438, 343 (2005).

[27] H.-P. Ren, M. S. Baptista, and C. Grebogi, Phys. Rev. Lett. 110, 184101 (2013).

[28] E. A. Sobakinskaya, A. L. Pankratov, and V. L. Vaks, Phys. Lett. A 376, 265 (2012).

[29] E. Sobakinskaya, V. L. Vaks, N. Kinev, M. Ji, M. Y. Li, H. B. Wang, and V. P. Koshelets, J. Phys. D: Appl. Phys. 50, 035305 (2017).

[30] V. L. Vaks, E. G. Domracheva, M. B. Chernyaeva, S. I. Pripolzin, L. S. Revin, I. V. Tretjyakov, V. A. Anfertev, and A. A. Yablokov, Opt. Quant. Electron. 49, 239 (2017).

[31] H. C. Sun, Z. B. Yang, N. V. Kinev, O. S. Kiselev, Y. Y. Lv, Y. Huang, L. Y. Hao, X. J. Zhou, M. Ji, X. C. Tu, C. H. Zhang, J. Li, F. Rudau, R. Wieland, J. S. Hampp, O. Kizilaslan, D. Koelle, B. B. Jin, J. Chen, L. Kang, W. W. Xu, R. Kleiner, V. P. Koshelets, H. B. Wang, and P. H. Wu, Phys. Rev. Appl. 8, 054005 (2017).

[32] V. Fischer, A closer look at security in random number generators design, in Constructive Side-Channel Analysis and Secure Design, edited by W. Schindler and S. A. Huss, Lecture Notes in Computer Science Vol. 7275 (Springer, Berlin, 2012), pp. 167-182.

[33] B. Qi, Y.-M. Chi, H.-K. Lo, and L. Qian, Opt. Lett. 35, 312 (2010).
[34] T. Symul, S. M. Assad, and P. K. Lam, Appl. Phys. Lett. 98, 231103 (2011).

[35] A. E. Ivanova, S. A. Chivilikhin, and A. V. Gleim, J. Phys.: Conf. Ser. 917, 062008 (2017).

[36] C. Gabriel, C. Wittmann, D. Sych, R. F. Dong, W. Mauerer, U. L. Andersen, C. Marquardt, and G. Leuchs, Nat. Photonics 4, 711 (2010).

[37] B. Sanguinetti, A. Martin, H. Zbinden, and N. Gisin, Phys. Rev. X 4, 031056 (2014).

[38] J.-L. Danger, S. Guilley, and P. Hoogvorst, Microelectron. J. 40, 1650 (2009).

[39] M. Sciamanna and K. A. Shore, Nat. Photonics 9, 151 (2015).

[40] A. Uchida, K. Amano, M. Inoue, K. Hirano, S. Naito, H. Someya, I. Oowada, T. Kurashige, M. Shiki, S. Yoshimori, K. Yoshimura, and P. Davis, Nat. Photonics 2, 728 (2008).

[41] I. Reidler, Y. Aviad, M. Rosenbluh, and I. Kanter, Phys. Rev. Lett. 103, 024102 (2009).

[42] I. Kanter, Y. Aviad, I. Reidler, E. Cohen, and M. Rosenbluth, Nat. Photonics 4, 58 (2010).

[43] B. A. Huberman, J. P. Crutchfield, and N. H. Packard, Appl. Phys. Lett. 37, 750 (1980).

[44] N. F. Pedersen and A. Davidson, Appl. Phys. Lett. 39, 830 (1981).

[45] V. N. Gubankov, K. I. Konstantinyan, V. P. Koshelets, and G. A. Ovsyannikov, IEEE Trans. Magn. 19, 637 (1983).

[46] R. L. Kautz and R. Monaco, J. Appl. Phys. 57, 875 (1985).

[47] Yu. M. Shukrinov, A. E. Botha, S. Yu. Medvedeva, M. R. Kolahchi, and A. Irie, Chaos 24, 033115 (2014).

[48] A. E. Botha, Yu. M. Shukrinov, and M. R. Kolahchi, Chaos, Solitons Fractals 48, 32 (2013).

[49] Yu. M. Shukrinov, H. Azemtsa-Donfack, and A. E. Botha, JETP Lett. 101, 251 (2015).

[50] A. E. Botha, Yu. M. Shukrinov, S. Yu. Medvedeva, and M. R. Kolahchi, J. Supercond. Novel Magn. 28, 349 (2015).

[51] A. L. Pankratov, E. V. Pankratova, V. A. Shamporov, and S. V. Shitov, Appl. Phys. Lett. 110, 112601 (2017).

[52] A. V. Ustinov, H. Kohlstedt, and P. Henne, Phys. Rev. Lett. 77, 3617 (1996).

[53] T. Nagatsuma, K. Enpuku, F. Irie, and K. Yoshida, J. Appl. Phys. 54, 3302 (1983).

[54] V. P. Koshelets, S. V. Shitov, P. N. Dmitriev, A. B. Ermakov, L. V. Filippenko, V. V. Khodos, V. L. Vaks, A. M. Baryshev, P. R. Wesselius, and J. Mygind, Phys. C (Amsterdam, Neth.) 367, 249 (2002).

[55] D. R. Gulevich and F. V. Kusmartsev, Phys. Rev. Lett. 97, 017004 (2006).

[56] D. R. Gulevich and F. V. Kusmartsev, Supercond. Sci. Technol. 20, S60 (2007).

[57] D. R. Gulevich and F. V. Kusmartsev, New J. Phys. 9, 59 (2007).

[58] D. R. Gulevich, M. Gaifullin, O. E. Kusmartseva, F. V. Kusmartsev, and K. Hirata, Phys. C (Amsterdam, Neth.) 468, 1903 (2008).

[59] A. L. Pankratov, A. S. Sobolev, V. P. Koshelets, and J. Mygind, Phys. Rev. B 75, 184516 (2007).

[60] D. R. Gulevich, P. N. Dmitriev, V. P. Koshelets, and F. V. Kusmartsev, Nanosystems: Phys. Chem. Math. 4, 507 (2013).

[61] P. N. Dmitriev, I. L. Lapitskaya, L. V. Filippenko, A. B. Ermakov, S. V. Shitov, G. V. Prokopenko, S. A. Kovtonyuk, and V. P. Koshelets, IEEE Trans. Appl. Supercond. 13, 107 (2003). 
[62] M. Yu. Torgashin, V. P. Koshelets, P. N. Dmitriev, A. B. Ermakov, L. V. Filippenko, and P. A. Yagoubov, IEEE Trans. Appl. Supercond. 17, 379 (2007).

[63] Nb-AlN-NbN technology [61,62] allows us to achieve higher radiation frequencies as compared to $\mathrm{Nb}-\mathrm{AlO}_{x}-\mathrm{Nb}$ junctions. The advantage of using Nb-AlN-NbN as opposed to NbN$\mathrm{AlN}-\mathrm{NbN}$ where both electrodes are made of $\mathrm{NbN}$ is achieving a better barrier quality. More specifically, while using the $\mathrm{Nb}$-AlN-NbN junction we can achieve a normal to subgap resistance ratio $R_{n} / R_{j}$ of about 30; the resistance ratio $R_{n} / R_{j}$ we could achieve for NbN-AlN-NbN contacts is limited to 10 .

[64] V. P. Koshelets, S. V. Shitov, A. V. Shchukin, L. V. Filippenko, J. Mygind, and A. V. Ustinov, Phys. Rev. B 56, 5572 (1997).

[65] The formula $V=V_{g} / 3$ comes from the fundamental Josephson relation $\hbar \omega=2 e V$ and the relation $e V+\hbar \omega=\Delta_{1}+\Delta_{2}$ for photon-assisted tunneling of quasiparticles.

[66] N. R. Werthamer, Phys. Rev. 147, 255 (1966).

[67] See Supplemental Material at http://link.aps.org/supplemental/ 10.1103/PhysRevB.99.060501 for the data obtained for two more experimental samples of TFFO, details of our numerical model, and analysis of the chaos generation mechanism.

[68] A. B. Ermakov, S. V. Shitov, A. M. Baryshev, V. P. Koshelets, and W. Luinge, IEEE Trans. Appl. Supercond. 11, 840 (2001).

[69] A. I. Larkin and Yu. N. Ovchinnikov, Sov. Phys. JETP 24, 1035 (1967).

[70] A. B. Zorin, I. O. Kulik, K. K. Likharev, and J. R. Schrieffer, Sov. J. Low Temp. Phys. 5, 537 (1979).

[71] A. A. Odintsov, V. K. Semenov, and A. B. Zorin, IEEE Trans. Magn. 23, 763 (1987).

[72] D. R. Gulevich, V. P. Koshelets, and F. V. Kusmartsev, Phys. Rev. B 96, 024515 (2017).

[73] D. R. Gulevich, L. V. Filippenko, and V. P. Koshelets, J. Low Temp. Phys., (2018), doi: 10.1007/s10909-018-2106-x.

[74] D. R. Gulevich, arXiv:1809.04706.
[75] MiTMoJCo (Microscopic tunneling model for Josephson contacts) is $\mathrm{C}$ and Python code is available under the GNU General Public License v3.0 at https://github.com/drgulevich/mitmojco.

[76] W. Bangerth, R. Hartmann, and G. Kanschat, ACM Trans. Math. Softw. 33, 24 (2007).

[77] D. Arndt, W. Bangerth, D. Davydov, T. Heister, L. Heltai, M. Kronbichler, M. Maier, J.-P. Pelteret, B. Turcksin, and D. Wells, J. Numer. Math. 25, 137 (2017).

[78] Open source finite element library deal.II, http://www.dealii. org.

[79] C. Geuzaine and J.-F. Remacle, Int. J. Numer. Meth. Eng. 79, 1309 (2009).

[80] G. Benettin, L. Galgani, and J.-M. Strelcyn, Phys. Rev. A 14, 2338 (1976).

[81] For this, we extend dimensionality of the Lyapunov vector to hold information about the previous evolution. During Benettin's successive renormalizations of perturbation vectors along the given direction we also renormalize the memory variables.

[82] J.-P. Eckmann, Rev. Mod. Phys. 53, 643 (1981).

[83] M. G. Li, J. Yuan, N. Kinev, J. Li, B. Gross, S. Guénon, A. Ishii, K. Hirata, T. Hatano, D. Koelle, R. Kleiner, V. P. Koshelets, H. B. Wang, and P. Wu, Phys. Rev. B. 86, 060505(R) (2012).

[84] O. Kiselev, M. Birk, A. Ermakov, L. Filippenko, H. Golstein, R. Hoogeveen, N. Kinev, B. van Kuik, A. de Lange, G. de Lange, P. Yagoubov, and V. Koshelets, IEEE Trans. Appl. Supercond. 21, 612 (2011).

[85] G. de Lange, D. Boersma, J. Dercksen, P. Dmitriev, A. B. Ermakov, L. V. Filippenko, H. Golstein, R. W. M. Hoogeveen, L. de Jong, A. V. Khudchenko, N. V. Kinev, O. S. Kiselev, B. van Kuik, A. de Lange, J. van Rantwijk, A. S. Sobolev, M. Yu. Torgashin, E. de Vries, P. A. Yagoubov, and V. P. Koshelets, Supercond. Sci. Technol. 23, 045016 (2010). 